INPLASY

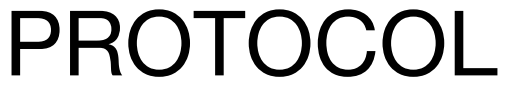

To cite: Li et al. Hyperbaric oxygen therapy for spinal cord injury: A systematic review and meta-analysis. Inplasy protocol 2020100084. doi:

10.37766/inplasy2020.10.0084

Received: 22 October 2020

Published: 22 October 2020

Corresponding author:

Tong Li

litong@stu.cdutcm.edu.cn

Author Affiliation:

Hospital of Chengdu

University of Traditional

Chinese Medicine

Support: The National Key

Research.

Review Stage at time of this submission: The review has not yet started.

Conflicts of interest:

None.

\section{Hyperbaric oxygen therapy for spinal cord injury: A protocol for systematic review and meta-analysis}

Li, T1; Wang, Y2; Feng, C3; Li, Q4; Ran, Q5; Chen, B6; Yu, Y7; Jiang, L8; Fan, $X^{9}$.

Review question / Objective: P: spinal cord injury; I:Hyperbaric oxygen therapy; C:Conventional treatments such as surgery, drugs and rehabilitation physiotherapy; 0:1.Primary outcomes: (1)American Spinal Injury Association(ASIA) Score: Includes American Spinal Injury Association Impairment Scale (AIS) and Asia Motor Score (AMS), (2)Frankel Classification Grading System, (3)Functional Independence Measure (FIM), (4)Barthel Index; 2.Secondary outcomes: (1)Hospitalization Time, (2)Death Rate, (3)Incidence of Any Expected or Unexpected Adverse Event, (4)Hamilton Anxiety Rating Scale (HAM-A), (5)Hamilton Depression Rating Scale (HAM-D); S:RCT.

Condition being studied: Hyperbaric oxygen (HBO) therapy can prevent further spinal cord injury $(\mathrm{SCl})$ caused by spinal cord ischemia-reperfusion injury to the maximum extent, which has been reported increasingly in recent years. However its security and effectiveness still lack of highquality evidence-based medical evidence. In this study, we will perform a systematic review of previously published randomized controlled trials to evaluate the efficacy and safety of HBO therapy for SCI.

INPLASY registration number: This protocol was registered with the International Platform of Registered Systematic Review and Meta-Analysis Protocols (INPLASY) on 22 October 2020 and was last updated on 29 October 2020 (registration number INPLASY2020100084).

\section{INTRODUCTION}

Review question / Objective: P: spinal cord injury; I:Hyperbaric oxygen therapy; C:Conventional treatments such as surgery, drugs and rehabilitation physiotherapy; O:1.Primary outcomes: (1)American Spinal Injury Association(ASIA) Score: Includes American Spinal Injury Association Impairment Scale (AIS) and Asia Motor 
Score (AMS), (2)Frankel Classification Grading System, (3)Functional Independence Measure (FIM), (4)Barthel Index; 2. Secondary outcomes: (1)Hospitalization Time, (2)Death Rate, (3) Incidence of Any Expected or Unexpected Adverse Event, (4)Hamilton Anxiety Rating Scale (HAM-A), (5)Hamilton Depression Rating Scale (HAM-D); S:RCT.

Condition being studied: Hyperbaric oxygen (HBO) therapy can prevent further spinal cord injury (SCl) caused by spinal cord ischemia-reperfusion injury to the maximum extent, which has been reported increasingly in recent years. However its security and effectiveness still lack of highquality evidence-based medical evidence. In this study, we will perform a systematic review of previously published randomized controlled trials to evaluate the efficacy and safety of HBO therapy for SCI.

\section{METHODS}

Participant or population: Any adult patients( $\geq 18$ years) diagnosed with $\mathrm{SCl}$ will be included in this study regardless their ethnicity, sex, age, and the length and severity of disease.

Intervention: The experimental group is hyperbaric oxygen therapy combined with conventional treatments such as surgery, drugs and rehabilitation physiotherapy.

Comparator: The control group received conventional treatments such as surgery, drugs and rehabilitation physiotherapy.

Study designs to be included: Randomized controlled trials (RCTs) will be included in this systematic review regardless of publication status and language.

Eligibility criteria: Randomized controlled trials (RCTs) will be included in this systematic review regardless of publication status and language.

Information sources: PubMed, Embase, Cochrane Library, Web of Science, China National Knowledge Infrastructure (CNKI), Chinese Science and Technology Periodical
Database (VIP), Wanfang database and Chinese Biomedical Literature Database (CBM).

Main outcome(s): (1)American Spinal Injury Association(ASIA) Score: Includes American Spinal Injury Association Impairment Scale (AIS) and Asia Motor Score (AMS) (2)Frankel Classification Grading System (3)Functional Independence Measure (FIM) (4)Barthel Index.

Additional outcome(s): (1)Hospitalization Time (2)Death Rate (3)Incidence of Any Expected or Unexpected Adverse Event (4)Hamilton Anxiety Rating Scale (HAM-A) (5)Hamilton Depression Rating Scale (HAMD).

Quality assessment / Risk of bias analysis: Two contributors will perform quality assessments and review the risk of bias using the Cochrane Collaboration's risk-ofbias assessment method (v6)[36]. This scale includes 7 risk of bias items, and each will be described as low, unclear, or high risk. Data will be presented in the risk of bias graph. Discrepancies will be resolved or as required, by a third reviewer.

Strategy of data synthesis: We will employ RevMan 5.3 (Cochrane Community, London, UK) software to synthesize and analyze the data, and to perform a metaanalysis if possible. If acceptable heterogeneity is examined among included trials, we will conduct a meta-analysis in accordance with the few variations in study and patient characteristics, and few differences in treatments, controls, and outcomes. If considerable heterogeneity is identified, we will carry out subgroup analysis and sensitivity analysis to find out any possible sources of obvious heterogeneity. If it is impossible to undertake a meta-analysis, we will report study results as a narrative summary.

Subgroup analysis: We will observe the source of considerable heterogeneity by subgroup analysis based on variations in study and patient characteristics, study 
quality, different interventions, comparators, and outcomes.

Sensibility analysis: We will perform sensitivity analysis to test the robustness and satiability of conclusions by removing low quality trials, and trials with small sample size.

Country(ies) involved: China.

Keywords: Spinal Cord Injury, Hyperbaric Oxygen Therapy, Meta-Analysis, Protocol.

Contributions of each author:

Author 1 - Tong Li.

Author 2 - Yiran Wang.

Author 3 - Chaoqun Feng.

Author 4 - Qianchun Li.

Author 5 - Qiang Ran.

Author 6 - Botao Chen.

Author 7 - Yang Yu.

Author 8 - Leming Jiang.

Author 9 - Xiaohong Fan. 